\title{
Remodeling of Crossbridges Controls Peptidoglycan Cross- linking Levels in Bacterial Cell Walls
}

\author{
Sean E. Pidgeon, ${ }^{\dagger}$ Alexis J. Apostolos, ${ }^{\dagger}$ and Marcos M. Pires*, \\ ${ }^{1}$ Department of Chemistry, Lehigh University, Bethlehem, Pennsylvania 18015, United States \\ ${ }^{\dagger}$ Authors contributed equally to this work
}

\section{Supporting Information Placeholder}

\begin{abstract}
Cell walls are barriers found in almost all known bacterial cells. These structures establish a controlled interface between the external environment and vital cellular components. A primary component of cell wall is a highly crosslinked matrix called peptidoglycan (PG). PG crosslinking, carried out by transglycosylases and transpeptidases, is necessary for proper cell wall assembly. Transpeptidases, targets of $\beta$-lactam antibiotics, stitch together two neighboring PG stem peptides (acyl-donor and acyl-acceptor strands). We recently described a novel class of cellular PG probes that were processed exclusively as acyl-donor strands. Herein, we have accessed the other half of the transpeptidase reaction by developing probes that are processed exclusively as acyl-acceptor strands. The critical nature of the crossbridge on the PG peptide was demonstrated in live bacterial cells and surprising promiscuity in crossbridge primary sequence was found in various bacterial species. Additionally, acyl-acceptor probes provided insight into how chemical remodeling of the PG crossbridge (e.g., amidation) can modulate crosslinking levels, thus establishing a physiological role of PG structural variations. Together, the acyl-donor and -acceptor probes will provide a versatile platform to interrogate PG crosslinking in physiologically relevant settings.
\end{abstract}

Gram-positive pathogens continue to impose a significant global public health burden. ${ }^{1}$ Among these organisms, Enterococci are one of the leading causes of nosocomial infections. ${ }^{2}$ With the rise in antibiotic drug resistance, it has become clear that more bacterial targets are needed to improve the therapeutic options to fight Enterococci infections. Peptidoglycan (PG), an essential component of bacterial cell walls, is one of the most important targets of antibiotics. Disaccharides of $\mathrm{N}$-acetylglucosamine (GlcNAc) and $\mathrm{N}$-acetylmuramic acid (MurNAc) make up the basic PG unit, which is further decorated with a pentameric stem peptide (e.g., LAla-D-isoGlx-L-Lys-D-Ala-D-Ala). ${ }^{3-4}$ PG units are polymerized and crosslinked via transglycosylation and transpeptidation (TP) reactions that assemble the sugars and peptides, respectively. PG crosslinking is essential, and, therefore, molecules that block this reaction are traditionally valuable antibiotics (e.g., $\beta$-lactams and vancomycin) and continue to result in promising drug leads (e.g., teixobactin). ${ }^{5-7}$

Intense efforts have led to a greater understanding of the chemical makeup, mechanism of assembly, and essential proteins that construct the PG network. Moreover, there is some evidence that small structural modifications within the PG matrix can alter the overall plasticity and physical properties of the cell wall (e.g., resistance to lysozyme). ${ }^{8-10}$ Chemical composition of the stem peptide primary sequence can vary by the amidation/methylation of carboxylic groups, removal of amino acids, variation of amino acid character, and extensive alteration to the central lysine group. Yet, for the most part, their physiological impact

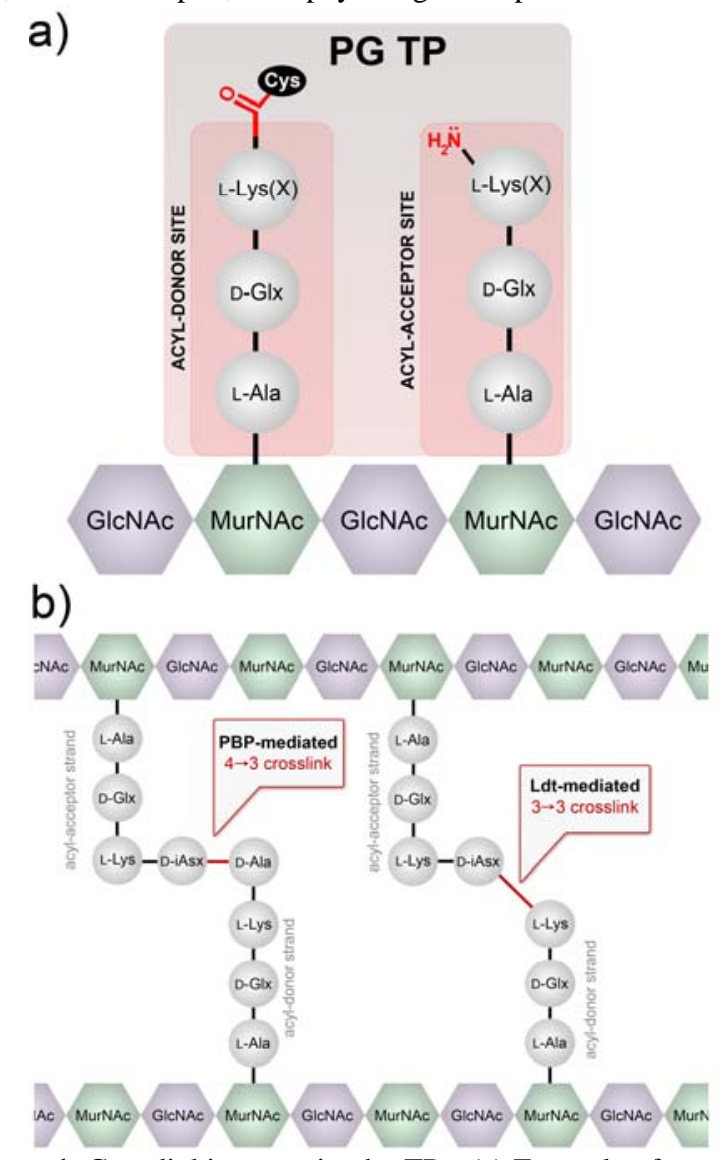

Figure 1. Crosslinking reaction by TPs. (a) Example of two binding sites (acyl-donor and -acceptor) at the intermediate step of the crosslinking reaction are depicted. The acyl-chain is covalently anchored on the TP, which is subsequently transferred to the amino group on the acyl-acceptor strand. $X$ represents the crossbridging amino acids. (b) Schematic of PG crosslinking in $E$. faecium showing the 2 types of crosslinks observed.

remains elusive. ${ }^{11-14}$

The $3^{\text {rd }}$ position with the PG stem peptide has defining structural characteristics. As an example, the difference of a carboxylic acid group on L-Lys verus meso-2,6-diaminopimelic acid ( $m$ DAP) has important implications in the activation of the human innate immune system. ${ }^{15}$ In Enterococcus faecium (E. faecium), the lysine sidechain is modified with D-iAsx while Enterococcus faecalis (E. faecalis) displays L-Ala-L-Ala. In addition, the nature of the crossbridge (the amino acids anchored onto the lysine residues) is critical based on its role in the nucleophilic step during 
a)
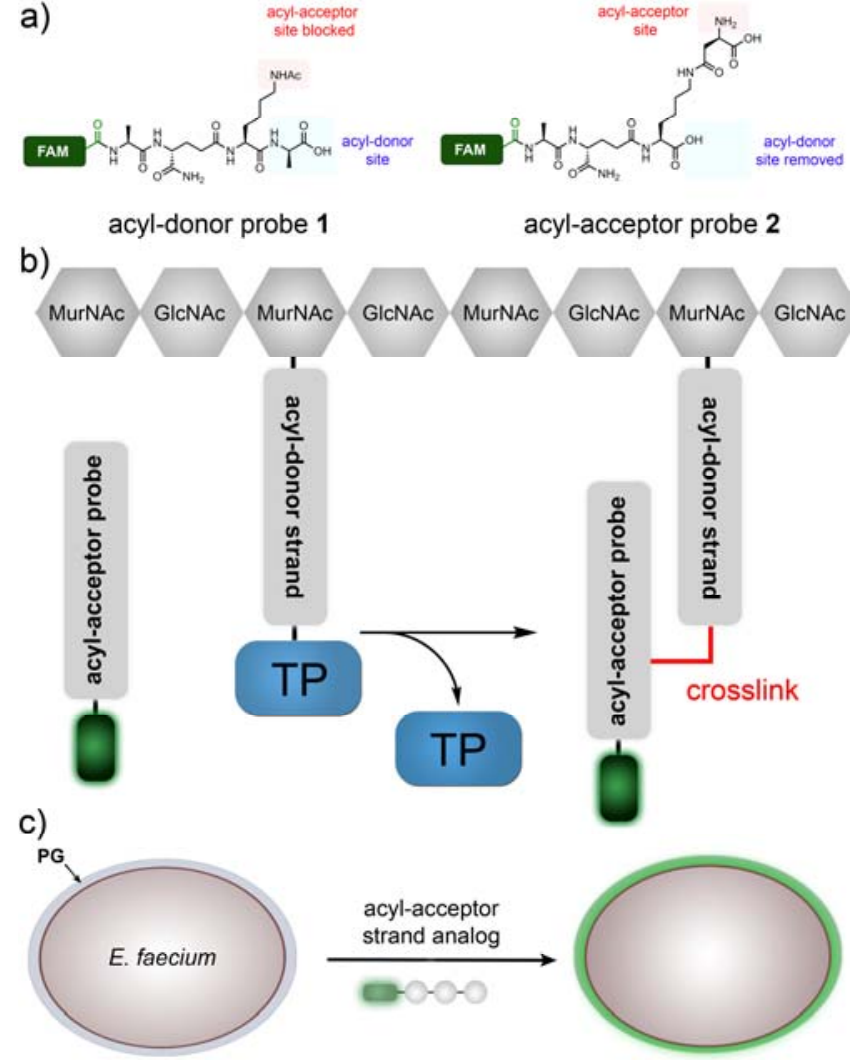

Figure 2. Design of tripeptide probes and the assay to assess acylacceptor strand in live cells. (a) Structure-based design of acyldonor and -acceptor probes. (b) Schematic diagram showing how the fluorescently tagged tripeptide probe gets covalently crosslinked into the bacterial PG scaffold. (c) Schematic of the whole cell analysis that can be accessed by the tripeptide probes.

crosslinking reactions. There are two main classes of PG crosslinking enzymes: D,D-TPs and L,D-TPs (Figure 1). Crosslinking by Penicillin Binding Protein (PBP) D,D-TPs generate 4-3 peptide crosslinks and L,D-TPs (Ldts) create 3-3 crosslinks within PG.

Fundamental understanding of PG crosslinking is key to define the processes that control PG structure and may provide novel drug targets. Single D-amino acid PG probes have spurred valuable insight into PG crosslinking in live bacterial cells. ${ }^{16-21}$ Furthermore, elegant prior in vitro studies using synthetic PG analogs have provided insight into the substrate structural requirements of TPs. ${ }^{12,}$ 22-25 However, fewer reports have described probes that decipher TP substrate requirements in live bacterial cells. A recent example of a synthetic L-Lys-D-Ala-D-Ala stem peptide mimic led to the demonstration of extraseptal TP activity in Staphylococcus aureus (S. aureus). ${ }^{26}$ Likewise, we recently reported the use of synthetic PG probes to establish crosslinking parameters based on the acyl-donor strand of Ldts. ${ }^{27}$ We have now built on those findings by developing complementary probes that selectively track and dissect acyl-acceptor strand processing by TPs in live bacterial cells.

We initially hypothesized that structural analogs of the two TP substrates could be developed to control active site loading. In designing our previous acyl-donor probe $\mathbf{1}$, we mimicked the stem peptide fragment as a tetrapeptide and blocked the nucleophilic site on the acyl-acceptor strand (Figure 2a). In this work, the acyl-acceptor specific probe $\mathbf{2}$ was designed by installing the acylacceptor fragment (crossbridging D-iAsx in the case of $E$. faecium) and removing the terminal fragment recognized by the acyl-donor site (terminal D-Ala and/or D-Ala-D-Ala). Therefore, PG probes with the basic scaffold of tripeptide 2 should act

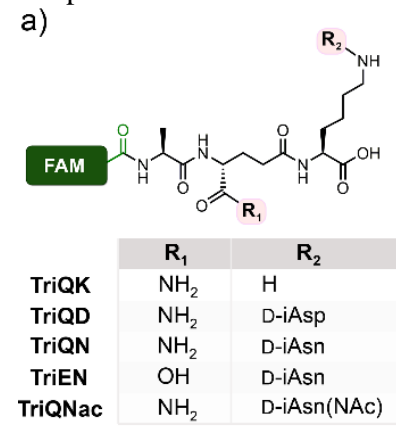

b)
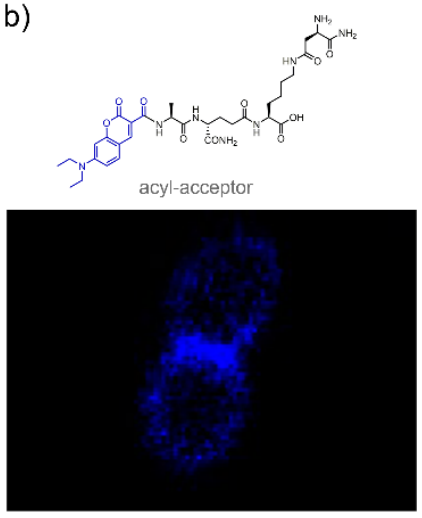

Figure 3. Crosslinking of tripeptide probes in live E. faecium cells. (a) Chemical series tripeptide probes based on the modifications at the D-iGlx and lysine sidechain; FAM = carboxyfluorescein. Flow cytometry analysis of E. faecium (D344s) treated overnight with $100 \mu \mathrm{M}$ of tri-peptide probes. Data are represented as mean $+\mathrm{SD}(\mathrm{n}=3)$. (b) Confocal microscopy of E. faecium cells labeled with the designated probes $(100 \mu \mathrm{M})$ for $5 \mathrm{~min}$. Scale bar $=2 \mu \mathrm{m}$.

solely as an acyl-acceptor strand. To track incorporation into the PG, a fluorescent handle was added to the $N$-terminus of the stem tripeptide analogs. Incubation of live bacterial cells with tripeptide probes is expected to result in their covalent incorporation into the PG scaffold by TPs (Figure $2 \mathbf{b}$ ). Crosslinking can then be readily quantified and analyzed using standard fluorescence-based techniques, thus providing a mode to interrogate how primary sequences of the acyl-acceptor strand modulate PG crosslinking (Figure 2c).

At first, we synthesized a panel of tripeptide probes that were designed to investigate acyl-acceptor strand recognition in live $E$. faecium (Figure 3). D-iAsx is the canonical crossbridge amino acid in E. faecium, where a nucleophilic attack by the sidechain $N$-terminus nitrogen leads to formation of PG crosslinks. All probes were synthesized using standard solid phase peptide chemistry and included a carboxy-fluorescein tag (FAM). E. faecium cells were treated with individual probes and cellular fluorescence levels were measured after overnight incubation. The first two stem peptide mimics, TriQK and TriQD, were designed to test the role of crossbridging amino acids in the acyl-capture step of TP. Our results showed that when cells were treated with TriQK, which lacks the crossbridging amino acid, fluorescence levels were near background (Figure 3a). Introduction of the crossbridge D-iAsp (TriQD) led to $\sim 7$ - fold fluorescence increase over background. These results provide in vivo evidence for the importance of the crossbridge for robust crosslinking of the PG scaffold and suggest that the enzyme responsible for D-iAsp installation, $\mathrm{Asl}_{\mathrm{fm}}$, ${ }^{28}$ is a potential narrow-spectrum drug target. 
Given that only approximately $60 \%$ of the lysines are modified with D-iAsx in E. faecium $^{29}$, and that we have evidence that unmodified lysine residues do not participate in TP reactions as acyl acceptors, these results also suggest a mode of controlling PG crosslinking based on $\mathrm{Asl}_{\mathrm{fm}}$ processing of PG precursors in the cytosol of cells prior to exposure to TPs.

We next evaluated how chemical remodeling of the acylacceptor strand may influence TP crosslinking in E. faecium. While the sidechain of the $3^{\text {rd }}$ position lysine is initially loaded with a D-iAsp crossbridge, its carboxylic acid can be biochemically amidated to generate D-iAsn crossbridges to varying levels in E. faecium. ${ }^{29}$ The physiological and TP-processing consequences of D-iAsp amidation remain unresolved due to confounding chemical re-arrangements (e.g., deamidation of D-iAsn and cyclization) during PG isolation and analysis. ${ }^{29}$ Instead, we hypothesized that we could establish the role of amidation using synthetic analogs in live bacteria. Strikingly, treatment of E. faecium with TriQN resulted in considerably higher crosslinking levels relative to TriQD (Figure 3). These results suggest that amidation of D-iAsp within the acyl-acceptor strand play a critical role in controlling PG crosslinking levels.

We then analyzed the role of amidation in the $2^{\text {nd }}$ position DiGlu in the crosslinking step. Recent genetic analyses revealed the essential nature of the genes encoding the enzymes that carry out the amidation of D-iGlu to D-iGln in S. aureus and Streptococcus pneumoniae. ${ }^{30-31}$ Subsequent in vitro characterization suggested that lack of D-iGlu amidation impairs PG crosslinking. ${ }^{11,23}$ Yet, it remained unresolved whether D-iGlu amidation impacts processing at the acyl-donor, acyl-acceptor, or both sites. We recently demonstrated that amidation of D-iGlu is critical for the acyldonor strand for both classes of TPs. ${ }^{27}$ To test these concepts in the acyl-acceptor substrate, E. faecium cells were treated with TriEN. In sharp contrast to the D-iGln variant (TriQN), cellular fluorescence levels were significantly lower in cells treated with the D-iGlu variant (TriEN). Together, our data point to a threelevel biochemical control (addition of crossbridge, D-iGlu to DiGln, and D-iAsp to D-iAsn) of the

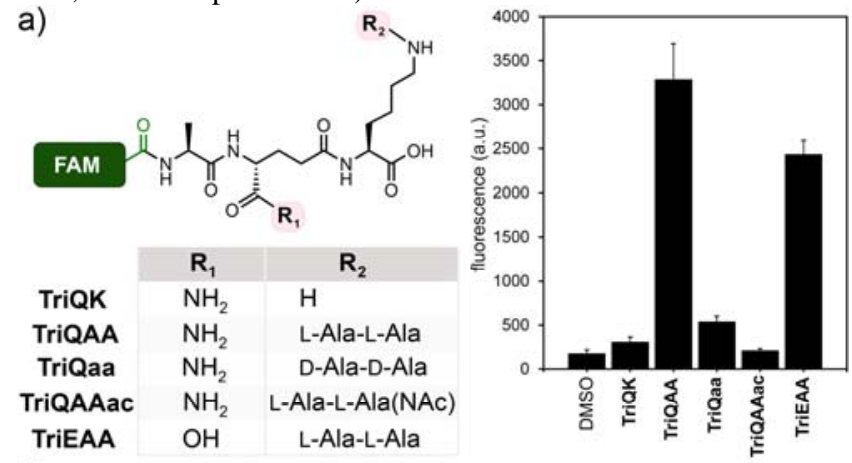

b)

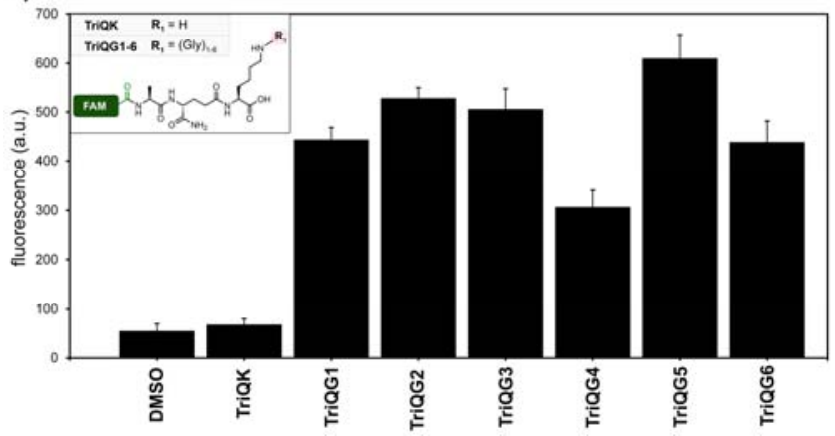

Figure 4. Crosslinking of tripeptide probes in live E. faecalis and $S$. aureus cells. Chemical series tripeptide probes based corresponding to the stem peptide of (a) E. faecalis and (b) S. aureus.
Flow cytometry analysis of cells treated overnight with $100 \mu \mathrm{M}$ of tri-peptide probes. Data are represented as mean $+\mathrm{SD}(\mathrm{n}=3)$.

acyl-acceptor substrate structure that likely combine to tune crosslinking level in E. faecium.

To confirm the incorporation of TriQN into the PG scaffold, metabolically labeled PG were digested and the resulting fragments analyzed (data not shown). Moreover, we demonstrated that the crossbridge of TriQN is, in fact, the acyl-acceptor site by building a control probe (TriQNAc) in which the nucleophilic amino group is acetylated to block acyl-capture. Fluorescence levels for cells treated with TriQNAc were near background, a result that is consistent with the site of acyl-capture. Confocal microscopy was then used to delineate the incorporation of the probes within the PG scaffold of live cells. For these experiments, a short pulse labeling step was performed with the acyl-acceptor probe modified with a blue fluorophore and the acyl-donor probe modified with a red fluorophore (Figure 3b). From our results, it is clear that there is some overlap between PG incorporation of these probes and we established that the septal region is the primary site of PG incorporation.

To extend our results to another type of Enterococci, acylacceptors probes were adopted for $E$. faecalis. In the case of $E$. faecalis, ligases BppA1 and BppA2 are responsible for transferring L-Ala from Ala- $t$ RNA to the first and $2^{\text {nd }}$ positions of the $\varepsilon^{-}$ amino group of lysine of PG precursors, respectively. ${ }^{32}$ We synthesized TriQAA, which mimics the crossbridge in E. faecalis, to initially test acyl-acceptor processing (Figure 4a). Incubation of E. faecalis cells with TriQAA led to a $\sim 10.5$ fold increase over the unmodified lysine side chain TriQK, thus again demonstrating the necessity for a crossbridging unit for proper acyl capture strand in Enterococci PG crosslinking. Inverting the stereochemistry of the crossbridge in TriQAA to TriQaa also led to baseline fluorescence, indicating that TPs are stereospecific at the acylacceptor position. Acetylation of the E. faecalis probe, TriQAAac, which blocks acyl-acceptor nucleophilic site, led to baseline fluorescence levels. This finding is consistent with the amino group of the terminal L-alanine as the nucleophile for covalent crosslinking into the PG scaffold. Surprisingly, lack of amidation at the second position (TriEAA) only resulted in a $\sim 1.3$-fold decrease in fluorescence, showing that amidation may not be an absolute requirement for the acyl-acceptor recognition in E. faecalis.

We next turned our attention to the problematic human pathogen $S$. aureus. In $S$. aureus, peptidyl transferases FemX, FemA, and FemB catalyze the stepwise addition of five glycines to the lysine residue on the $3^{\text {rd }}$ position of the PG precursor. ${ }^{33-35}$ Recently, Walker and co-workers isolated PG precursors corresponding to one, three, and five glycine units

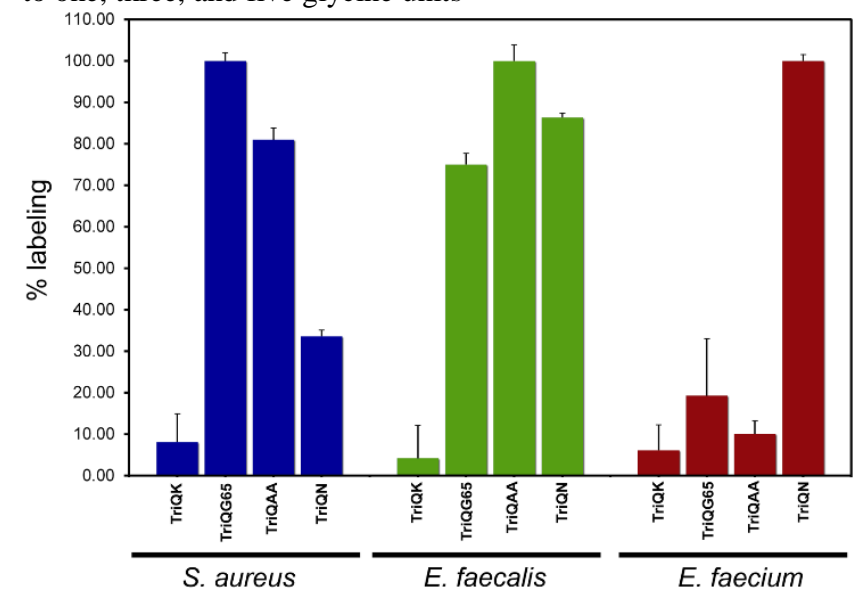


Figure 5. Crossbridge preferences across bacterial species. Flow cytometry analysis of cells treated overnight with $100 \mu \mathrm{M}$ of tripeptide probes. Data are represented as mean $+\mathrm{SD}(\mathrm{n}=3)$.

and showed in vitro that PBP transpeptidases have varying levels of preference for the length of the glycine chain. ${ }^{12}$ We built a library of tripeptide $S$. aureus probes that complement their approach by assessing the acyl-acceptor preference in live bacterial cells. We synthesized TriQG1-6 (Figure 4b), corresponding to glycine chains from 1 to 6 units, and measured their incorporation into the PG scaffold of $S$. aureus cells. Consistent with our previous results, the absence of glycine modification (TriQK) resulted in background levels of PG incorporation. Interestingly, addition of a single glycine (TriQG1) led to $\sim 7$-fold increase in cellular fluorescence levels. These results are consistent with a previously reported viable $S$. aureus fem $A B$-null strain in which one glycine crossbridges were observed, albeit with severe crosslinking defects and impaired growth. ${ }^{35-36}$ Our results clearly demonstrate the physiological consequence of a lack of a single glycine unit and point to FemX is an particularly attractive target for therapeutic development, something that had been suggested but had not been observed in live bacterial cells due to the lethality of femXdeletion. ${ }^{37}$ Crossbridges of 2 to 5 glycine resulted in comparable labeling levels. Extending beyond the natural pentaglycine chain (TriQG6) did not significantly impact crosslinking, which suggests that there is a high level of flexibility by the acyl-acceptor strand in S. aureus.

Having individually established the structural preference of the acyl-acceptor strand for various bacteria, we next set out to examine how crossbridges are tolerated by TPs across different species. Because TPs are frequent targets of $\beta$-lactams and other potent antibiotics, these enzymes play central roles in the drug resistance among several classes of medically relevant bacteria. ${ }^{38}$ Moreover, mobile elements carrying TP genetic information can be a powerful modality for the acquisition of drug resistant phenotypes. The $m e c A$ gene underpinning the drug resistant phenotype of Methicillin-resistant Staphylococcus aureus (MRSA) has been proposed to originate from Staphylococcus sciuri (S. sciuri). ${ }^{39}$ Therefore, the ability of TPs to process diverse PG architectures may have implications on the potential of TP to cross polinate drug resistance phenotypes. As an example, it was recently shown that commensal streptococci can serve as a reservoir for PBPs that can cross pollinate drug resistant phenotypes. ${ }^{40}$

To investigate the tolerance of non-native crossbridges in PG crosslinking reactions, the three canonical crossbridges (TriQN, TriQAA, and TriQG5) were incubated with E. faecium, E. faecalis, and S. aureus. S. aureus showed some promiscuity in accepting both TriQAA and TriQN (Figure 5 and Figure SX for raw values) while still demonstrating some preference for pentaglycine crossbridge. Conversely, E. faecium showed strong preference for its natural acyl-acceptor strand TriQN. Treatment of E. faecium cells with TriQAA or TriQG5 led to background fluorescence levels. Strikingly, E. faecalis showed little preference in the acyl-acceptor side chain and labeled well with all three tripeptide probes. . These findings are consistent with previous in vitro studies that illustrated the promiscuity of $E$. faecalis TPs in accepting non-native crossbridges. ${ }^{41-42}$ Our results provide in vivo evidence that some bacterial strains possess a high degree of tolerance for exogenous crossbridges, which may ultimately provide a potentially viable drug resistance modality.

Targeting the enzymatic processes that control PG assembly is a powerful method of disabling bacterial pathogens. Overall, our results demonstrate the requirement of the crossbridge in acylacceptors for proper crosslinking by TPs in three different types of bacteria, thus reinforcing the concept that lysine modification is pivotal in tuning the PG crosslinking machinery. Future investigations will center on finding out how flexibility in PG crosslinking may promote the evolution of drug resistance. In developing a comprehensive structural analysis of both the acyl-donor and acceptor strands, we anticipate that these results may guide drug design and open new avenues of therapeutic modalities.

\section{ASSOCIATED CONTENT}

\section{Supporting Information}

Additional experimental details (methods, characterization and synthesis of peptide probes) and figures.

\section{AUTHOR INFORMATION}

\section{Corresponding Author}

map311@lehigh.edu

\section{Notes}

The authors declare no competing financial interests.

\section{ACKNOWLEDGMENT}

This study was supported by GM124893-01 (M.P).

\section{REFERENCES}

1. Andersson, D. I.; Hughes, D., Antibiotic resistance and its cost: is it possible to reverse resistance? Nat Rev Microbiol 2010, 8 (4), 260-71.

2. Arias, C. A.; Murray, B. E., The rise of the Enterococcus: beyond vancomycin resistance. Nat Rev Microbiol 2012, 10 (4), 266-78.

3. Vollmer, W.; Blanot, D.; de Pedro, M. A., Peptidoglycan structure and architecture. FEMS Microbiol Rev 2008, 32 (2), 149-67.

4. Silhavy, T. J.; Kahne, D.; Walker, S., The bacterial cell envelope. Cold Spring Harb Perspect Biol 2010, 2 (5), a000414.

5. Ling, L. L.; Schneider, T.; Peoples, A. J.; Spoering, A. L.; Engels, I.; Conlon, B. P.; Mueller, A.; Schaberle, T. F.; Hughes, D. E.; Epstein, S.; Jones, M.; Lazarides, L.; Steadman, V. A.; Cohen, D. R.; Felix, C. R.; Fetterman, K. A.; Millett, W. P.; Nitti, A. G.; Zullo, A. M.; Chen, C.; Lewis, K., A new antibiotic kills pathogens without detectable resistance. Nature 2015, 517 (7535), 455-9.

6. Breukink, E.; de Kruijff, B., Lipid II as a target for antibiotics. Nat Rev Drug Discov 2006, 5 (4), 321-32.

7. Bugg, T. D.; Braddick, D.; Dowson, C. G.; Roper, D. I., Bacterial cell wall assembly: still an attractive antibacterial target. Trends Biotechnol 2011, 29 (4), 167-73.

8. Wang, G.; Lo, L. F.; Forsberg, L. S.; Maier, R. J., Helicobacter pylori peptidoglycan modifications confer lysozyme resistance and contribute to survival in the host. MBio 2012, 3 (6), e00409-12.

9. Juan, C.; Torrens, G.; Barcelo, I. M.; Oliver, A., Interplay between Peptidoglycan Biology and Virulence in Gram-Negative Pathogens. Microbiol Mol Biol Rev 2018, 82 (4).

10. Vijayrajratnam, S.; Pushkaran, A. C.; Balakrishnan, A.; Vasudevan, A. K.; Biswas, R.; Mohan, C. G., Bacterial peptidoglycan with amidated meso-diaminopimelic acid evades NOD1 recognition: an insight into NOD1 structure-recognition. Biochem J 2016, 473 (24), 45734592 .

11. Zapun, A.; Philippe, J.; Abrahams, K. A.; Signor, L.; Roper, D. I.; Breukink, E.; Vernet, T., In vitro reconstitution of peptidoglycan assembly from the Gram-positive pathogen Streptococcus pneumoniae. ACS Chem Biol 2013, 8 (12), 2688-96.

12. Srisuknimit, V.; Qiao, Y.; Schaefer, K.; Kahne, D.; Walker, S., Peptidoglycan Cross-Linking Preferences of Staphylococcus aureus Penicillin-Binding Proteins Have Implications for Treating MRSA Infections. J Am Chem Soc 2017, 139 (29), 9791-9794.

13. Morlot, C.; Straume, D.; Peters, K.; Hegnar, O. A.; Simon, N.; Villard, A. M.; Contreras-Martel, C.; Leisico, F.; Breukink, E.; GravierPelletier, C.; Le Corre, L.; Vollmer, W.; Pietrancosta, N.; Havarstein, L. S.; Zapun, A., Structure of the essential peptidoglycan amidotransferase 
MurT/GatD complex from Streptococcus pneumoniae. Nat Commun 2018, 9 (1), 3180.

14. Kumar, J. K., Lysostaphin: an antistaphylococcal agent. Appl Microbiol Biotechnol 2008, 80 (4), 555-61.

15. Franchi, L.; Warner, N.; Viani, K.; Nunez, G., Function of Nod-like receptors in microbial recognition and host defense. Immunol Rev 2009, 227 (1), 106-28.

16. Kuru, E.; Hughes, H. V.; Brown, P. J.; Hall, E.; Tekkam, S.; Cava, F.; de Pedro, M. A.; Brun, Y. V.; VanNieuwenhze, M. S., In Situ probing of newly synthesized peptidoglycan in live bacteria with fluorescent D-amino acids. Angew Chem Int Ed Engl 2012, 51 (50), 12519-23.

17. Kuru, E.; Tekkam, S.; Hall, E.; Brun, Y. V.; Van Nieuwenhze, M. S., Synthesis of fluorescent D-amino acids and their use for probing peptidoglycan synthesis and bacterial growth in situ. Nat Protoc 2015, 10 (1), 33-52.

18. Siegrist, M. S.; Whiteside, S.; Jewett, J. C.; Aditham, A.; Cava, F.; Bertozzi, C. R., (D)-Amino acid chemical reporters reveal peptidoglycan dynamics of an intracellular pathogen. ACS Chem Biol 2013, 8 (3), $500-5$.

19. Siegrist, M. S.; Swarts, B. M.; Fox, D. M.; Lim, S. A.; Bertozzi, C. R., Illumination of growth, division and secretion by metabolic labeling of the bacterial cell surface. FEMS Microbiol Rev 2015, 39 (2), 184-202.

20. Lebar, M. D.; May, J. M.; Meeske, A. J.; Leiman, S. A.; Lupoli, T. J.; Tsukamoto, H.; Losick, R.; Rudner, D. Z.; Walker, S.; Kahne, D., Reconstitution of peptidoglycan cross-linking leads to improved fluorescent probes of cell wall synthesis. J Am Chem Soc 2014, 136 (31), 10874-7.

21. Lupoli, T. J.; Tsukamoto, H.; Doud, E. H.; Wang, T. S.; Walker, S.; Kahne, D., Transpeptidase-mediated incorporation of D-amino acids into bacterial peptidoglycan. J Am Chem Soc 2011, 133 (28), 10748-51.

22. Lebar, M. D.; Lupoli, T. J.; Tsukamoto, H.; May, J. M.; Walker, S.; Kahne, D., Forming cross-linked peptidoglycan from synthetic gram-negative Lipid II. J Am Chem Soc 2013, 135 (12), 4632-5.

23. Ngadjeua, F.; Braud, E.; Saidjalolov, S.; Iannazzo, L.; Schnappinger, D.; Ehrt, S.; Hugonnet, J. E.; Mengin-Lecreulx, D.; Patin, D.; Etheve-Quelquejeu, M.; Fonvielle, M.; Arthur, M., Critical Impact of Peptidoglycan Precursor Amidation on the Activity of 1,d-Transpeptidases from Enterococcus faecium and Mycobacterium tuberculosis. Chemistry 2018, 24 (22), 5743-5747.

24. Welsh, M. A.; Taguchi, A.; Schaefer, K.; Van Tyne, D.; Lebreton, F.; Gilmore, M. S.; Kahne, D.; Walker, S., Identification of a Functionally Unique Family of Penicillin-Binding Proteins. J Am Chem Soc 2017, 139 (49), 17727-17730.

25. Gautam, S.; Kim, T.; Shoda, T.; Sen, S.; Deep, D.; Luthra, R.; Ferreira, M. T.; Pinho, M. G.; Spiegel, D. A., An Activity-Based Probe for Studying Crosslinking in Live Bacteria. Angew Chem Int Ed Engl 2015, 54 (36), 10492-6.

26. Pidgeon, S. E.; Apostolos, A. J.; Nelson, J. M.; Shaku, M.; Rimal, B.; Islam, M. N.; Crick, D. C.; Kim, S. J.; Pavelka, M. S.; Kana, B. D.; Pires, M. M., L,D-transpeptidase Specific Probe Reveals Spatial Activity of Peptidoglycan Crosslinking. ACS Chem Biol 2019.

27. Bellais, S.; Arthur, M.; Dubost, L.; Hugonnet, J. E.; Gutmann, L.; van Heijenoort, J.; Legrand, R.; Brouard, J. P.; Rice, L.; Mainardi, J. L., Aslfm, the D-aspartate ligase responsible for the addition of D-aspartic acid onto the peptidoglycan precursor of Enterococcus faecium. J Biol Chem 2006, 281 (17), 11586-94.

28. Patti, G. J.; Kim, S. J.; Schaefer, J., Characterization of the peptidoglycan of vancomycin-susceptible Enterococcus faecium. Biochemistry 2008, 47 (32), 8378-85.
29. Figueiredo, T. A.; Sobral, R. G.; Ludovice, A. M.; Almeida, J. M.; Bui, N. K.; Vollmer, W.; de Lencastre, H.; Tomasz, A., Identification of genetic determinants and enzymes involved with the amidation of glutamic acid residues in the peptidoglycan of Staphylococcus aureus. PLoS Pathog 2012, 8 (1), e1002508.

30. Liu, X.; Gallay, C.; Kjos, M.; Domenech, A.; Slager, J.; van Kessel, S. P.; Knoops, K.; Sorg, R. A.; Zhang, J. R.; Veening, J. W., Highthroughput CRISPRi phenotyping identifies new essential genes in Streptococcus pneumoniae. Mol Syst Biol 2017, 13 (5), 931.

31. Courtin, P.; Miranda, G.; Guillot, A.; Wessner, F.; Mezange, C.; Domakova, E.; Kulakauskas, S.; Chapot-Chartier, M. P., Peptidoglycan structure analysis of Lactococcus lactis reveals the presence of an L,D-carboxypeptidase involved in peptidoglycan maturation. J Bacteriol 2006, 188 (14), 5293-8.

32. Bouhss, A.; Josseaume, N.; Severin, A.; Tabei, K.; Hugonnet, J. E.; Shlaes, D.; Mengin-Lecreulx, D.; Van Heijenoort, J.; Arthur, M., Synthesis of the L-alanyl-L-alanine cross-bridge of Enterococcus faecalis peptidoglycan. J Biol Chem 2002, 277 (48), 45935-41.

33. Maidhof, H.; Reinicke, B.; Blumel, P.; Berger-Bachi, B.; Labischinski, H., femA, which encodes a factor essential for expression of methicillin resistance, affects glycine content of peptidoglycan in methicillin-resistant and methicillin-susceptible Staphylococcus aureus strains. J Bacteriol 1991, 173 (11), 3507-13.

34. Henze, U.; Sidow, T.; Wecke, J.; Labischinski, H.; BergerBachi, B., Influence of femB on methicillin resistance and peptidoglycan metabolism in Staphylococcus aureus. J Bacteriol 1993, 175 (6), 1612-20.

35. Monteiro, J. M.; Covas, G.; Rausch, D.; Filipe, S. R.; Schneider, T.; Sahl, H. G.; Pinho, M. G., The pentaglycine bridges of Staphylococcus aureus peptidoglycan are essential for cell integrity. Sci Rep 2019, $9(1), 5010$.

36. Stranden, A. M.; Ehlert, K.; Labischinski, H.; Berger-Bachi, B., Cell wall monoglycine cross-bridges and methicillin hypersusceptibility in a femAB null mutant of methicillin-resistant Staphylococcus aureus. J Bacteriol 1997, 179 (1), 9-16.

37. Tschierske, M.; Mori, C.; Rohrer, S.; Ehlert, K.; Shaw, K. J.; Berger-Bachi, B., Identification of three additional femAB-like open reading frames in Staphylococcus aureus. FEMS Microbiol Lett 1999, 171 (2), 97-102.

38. Waxman, D. J.; Strominger, J. L., Penicillin-binding proteins and the mechanism of action of beta-lactam antibiotics. Annu Rev Biochem 1983, 52, 825-69.

39. Wu, S.; Piscitelli, C.; de Lencastre, H.; Tomasz, A., Tracking the evolutionary origin of the methicillin resistance gene: cloning and sequencing of a homologue of mecA from a methicillin susceptible strain of Staphylococcus sciuri. Microb Drug Resist 1996, 2 (4), 435-41.

40. Jensen, A.; Valdorsson, O.; Frimodt-Moller, N.; Hollingshead, S.; Kilian, M., Commensal streptococci serve as a reservoir for betalactam resistance genes in Streptococcus pneumoniae. Antimicrob Agents Chemother 2015, 59 (6), 3529-40.

41. Magnet, S.; Arbeloa, A.; Mainardi, J. L.; Hugonnet, J. E.; Fourgeaud, M.; Dubost, L.; Marie, A.; Delfosse, V.; Mayer, C.; Rice, L. B.; Arthur, M., Specificity of L,D-transpeptidases from gram-positive bacteria producing different peptidoglycan chemotypes. J Biol Chem 2007, 282 (18), 13151-9.

42. Arbeloa, A.; Hugonnet, J. E.; Sentilhes, A. C.; Josseaume, N.; Dubost, L.; Monsempes, C.; Blanot, D.; Brouard, J. P.; Arthur, M., Synthesis of mosaic peptidoglycan cross-bridges by hybrid peptidoglycan assembly pathways in gram-positive bacteria. J Biol Chem 2004, 279 (40), 41546-56. 
bioRxiv preprint doi: https://doi.org/10.1101/840405; this version posted November 14, 2019. The copyright holder for this preprint (which was not certified by peer review) is the author/funder. All rights reserved. No reuse allowed without permission.

\section{SYNOPSIS TOC.}

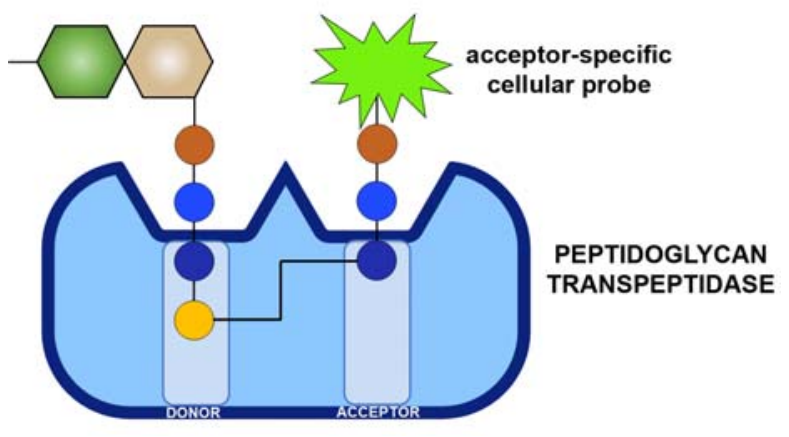

